Japanese Pychologial Rerant

1994, Vol 36. No, 3, 169-17?

Special lssue: Gestall Perception I

\title{
Perception of partly occluded objects in schizophrenic patients
}

\author{
MASAO YOKOTA \\ Department of Psychology, College of Humanities and Sciences, Nihon University, Sakurajosui, \\ Seragaya-ku. Tokvo 156
}

In drawing tests. schizophrenic patients could depict objects separately and in isolation, but not partly occluded objects. This finding may suggest that schizophrenic patients do not perceive partly occluded objects. To investigate this possibility, by means of Kanizsa's figures, 30 schizophrenic patients and 50 normal subjects were examined on how they perceive partly occluded objects. Only two schizophrenic patients perceived all stimuli as mosaics. The others perceived presented objects as completed behind another occluding object. The most frequent perceptual completions were based on the principle of 'good continuity' in schizophrenics, and on that of symmetry in normal subjects. This suggests that schizophrenic patients primarily process parts of an object rather than a whole object as normal subjects do. The principle of 'good continuity' thus appears to operate on more localized information than does the principle of symmetry in the test patterns in this experiment.

Key words: schizophrenia. perceptual completion, perceptual disorder.

Peculiar drawing features in schizophrenic patients have been found in many psychological drawing tests (Nakai, 1970; Mikami, 1979a, b; Miyazaki, Hujii, \& Kobayashi, 1987; Morita, 1989; Yokota, 1993). One of the most frequent features was isolation of objects in the Kusamura-Test, that asks subjects to depict themselves searching for a missing 500 yen coin in grass (Yokota, Yoda, Miyanaga, Takahashi, \& Machiyama, 1986), and in the Before-Behind Task, that asks subjects to depict a triangle behind a circle that has already been drawn (Yokota, 1992). Another frequent feature was overlapped drawings in the Separated Circles Task and the Conjointed Circles Task, that ask subjects to depict a triangle between two circles which has already been drawn

I would like to acknowledge gratefully Prof. Tadasu Oyama, Nihon University, for his helpful and valuable comments on the manuscript of this paper. I am indebted to Stephen Joseph Harding, Nihon University, for his helpful suggestions concerning English expression.
(Yokota, 1992). A less frequent feature was elimination of hidden lines in all tasks abovementioned. These findings suggest that schizophrenic patients can depict objects separately and in isolation but can not depict partly occluded objects.

In the Conjointed Circles Task, a line drawing of one circle partly occluding an other was used as the stimulus. This task demands the subject depict a triangle which is placed between two circles. It is necessary for the solution of the task for the subject to perceive the phenomenological relationship of "between two circles appearing to be at different distances from the observer". If perception of such a relationship does not occur, the meaning of "between two circles" is held to have not been understood by the subject. Schizophrenics' inability in drawing partly occluded objects might well be based on such a perceptual disorder.

When looking at a line drawing of one figure occluding another, most normal observers perceive the second figure as complete but behind the first. This phenomenon 
is known as 'amodal completion' (Kanizsa, 1979). However, no research has been done on amodal completion in schizophrenics. A line drawing of partly occluded objects is a representation of the figure-ground relationship between objects. Disorder of figureground perception in schizophrenic patients has been reported by some investigators. Weckowicz (1960) found that schizophrenic patients failed to perceive line drawings of familiar objects in which superimposed lines crossed. Crookes and Hutt (1970) reported that identification of simple geometrical figures embedded in complex designs were worse in schizophrenic patients than in neurotics. Weiner (1966) indicated that, in schizophrenic patients, figure and ground blurring was reflected by Rorschach WS responses which represent failure to differentiate space from the gray or colored aspects of the blots. These findings suggest disorder of segregation between figure and ground in schizophrenic patients. If this is true, does amodal completion appear in schizophrenic patients?

In this study, schizophrenic patients were examined on how they perceive line drawings of one figure partly occluding another, using Kanizsa's figures.

\section{Methods}

Subjects. The subjects were 30 schizophrenic inpatients (19 males and 11 females) of $\mathrm{H}$. Hospital in the Gunma Prefecture and 50 normal hospital employees ( 20 males and 30 females). All schizophrenic patients met the criteria for schizophrenia in DSM-III-R (American Psychiatric Association, 1987). The mean age of patients at the experiment was 34.6 years $(S D=5.2)$; Their mean age at first psychotic episode had been 22.4 years $(S D=5.2)$; Their mean length of the stay in hospital was 4.5 years $(S D=6.8)$. The mean age of normal subjects was 32.6 years $(S D=8.6)$.

Stimuli. Stimuli consisted of nine line drawings which had been illustrated as examples of amodal completion in Kanizsa (1979) (see Figure 1). According to Kanizsa, subjects perceive a complete figure behind a square presented in each line drawing. All stimuli were printed on a piece of paper in the same arrangement as in the nine stimuli shown in Figure 1.

Procedure. Each subject was shown nine stimuli simultaneously. Subjects were given the following instruction:

You will see a number of designs. These designs consist of partially covered figures. In every case a figure is hidden behind a square. If the square is removed, what kind of figure will you find? Add lines in each design and complete the figure that you expect to find.

After the instruction, subjects were given a pencil and asked to depict additional lines for each design. Schizophrenic patients were examined individually within an ordinal psychological test session and normal subjects were examined in groups of one to ten.

Classification of completions. According to the direction of lines drawn by the subjects at the crossing points, each completion was classified into the following five categories; a) mosaic, b) continuity of direction, c) symmetry, d) minimal distance and e) others (see Figure 2). In the mosaic category, the subject drew no partial occlusion. Amodal completion does not appear in this case. In the category of continuity of direction, the subject depicted a line preserving the continuity of direction at the crossing points. In the symmetry category, the subject completed a symmetrical figure that was behind a square. In the minimal distance category, the subject depicted the straight line that was the shortest distance between two crossing points. In the category of others, the subject depicted lines that could not be classified into the above-mentioned categories. 
Results

The results were classified according to the five categories of perceptual completion; their frequencies for each of the nine stimuli are shown in Table 1. It was found that more than two schizophrenic patients had depicted mosaics for each stimulus; the two of them always depicted mosaics for the test stimuli.

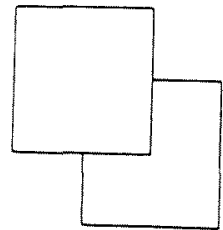

b
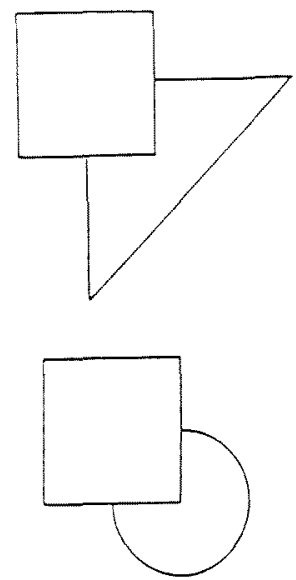

d

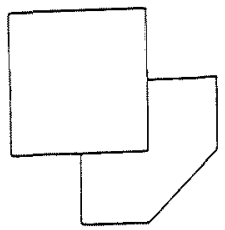

$e$

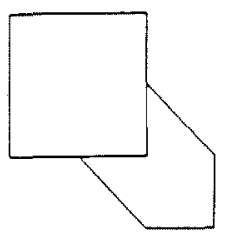

f

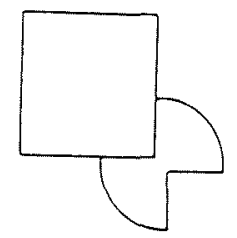

g

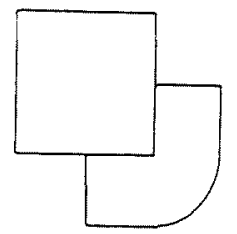

h

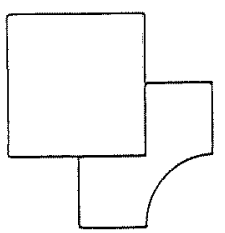

i

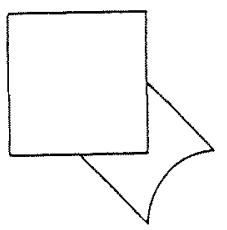

Figure 1. Stimuli used in this Experiment. 
response category is continuity of direction or symmetry. The most frequent completion was based on continuity of direction for all stimuli, except one (Stimulus e), in schizophrenic patients, and for six stimuli (Stimuli $\mathrm{a}, \mathrm{b}, \mathrm{c}, \mathrm{f}, \mathrm{g}$ and $\mathrm{h}$ ) in normal subjects. Symmetry was the most frequent for Stimuli $\mathrm{e}$ in both groups and for Stimuli $\mathrm{d}$ and $\mathrm{i}$ in normal subjects. Thus, most completions were based on either continuity of direction or symmetry in both groups.

Frequecies of categories were compared between schizophrenic patients and normal subjects. The frequency of continuity of direction in schizophrenic patients was significantly lower than in normal subjects for Stimulus c but significantly higher for Stimuli e and i. Frequency of symmetry in schizophrenic patients was significantly lower than in normal subjects for Stimuli d, $\mathrm{e}, \mathrm{g}$ and $\mathrm{h}$. These results indicate that schizophrenic patients complete more frequently by continuity of direction (except for Stimulus c) and complete less frequently by symmetry than normal subjects.

Table 1 also indicates that frequencies of continuity of direction for Stimuli $e$ and $i$ were lower than those for other Stimuli.

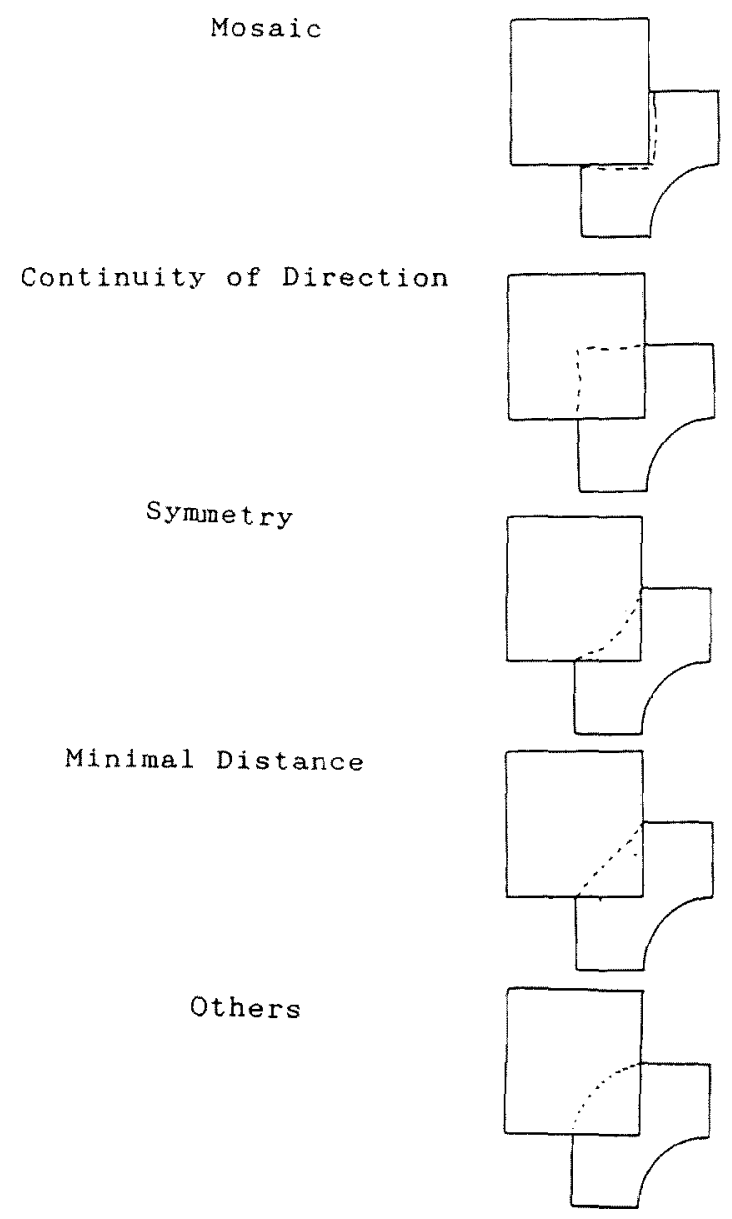

Figure 2. Examples of mosaic, continuity of direction, symmetry, minimal distance and other categories. 
Table 1

Frequencies of categories in schizophrenic patients and normals

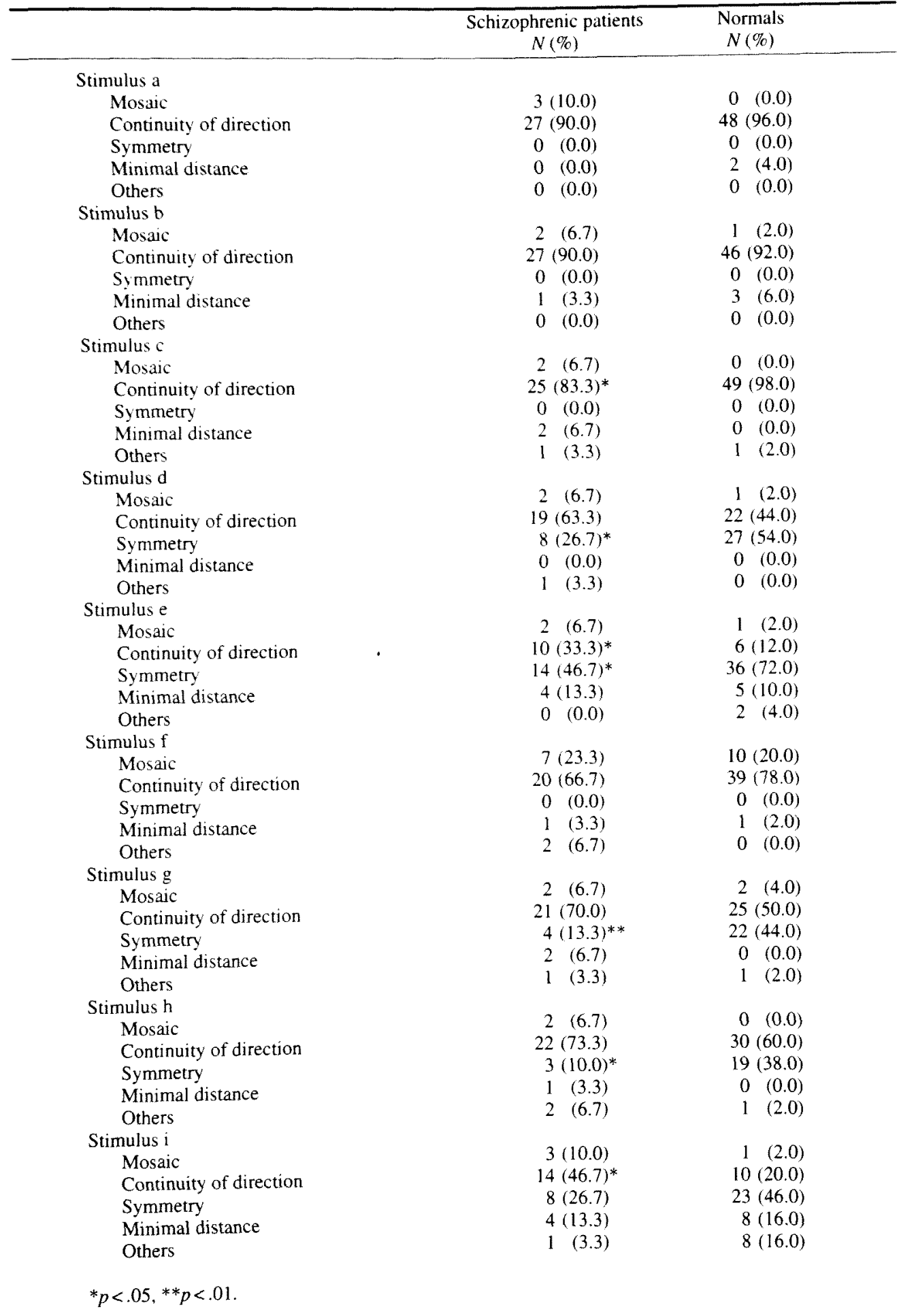


When we look at Stimuli e and $i$, we find that no closed design can result from continuity of direction only, whereas it can for the other stimuli. Therefore, it is necessary to reclassify the results for Stimuli e and i by another principle as in the examples shown in Figure 3. The depiction of 'minimal distance' in Figure 3 is not the same as that in Figure 2. A perpendicular line is added to parallel lines. Two parallel lines are connected by the principle of minimal distance. Therefore, this depiction can be included in the category of minimal distance. After the first classification, completions are reclassified for Stimuli e and $i$ according to the new principle illustrated by examples in Figure 3, and their frequencies are shown in Table 2.

Table 2 indicates that symmetry was the most frequent, and the frequencies of mosaic, symmetry, minimal distance and others categories were not significantly different between schizophrenic patients and normal subjects for both figures. Thus, the abovementioned tendency that symmetry was significantly less frequent in schizophrenic patients than in normal subjects (Table 1) had disappeared for Stimulus e. This means that schizophrenic patients' completions had two stages, that is, continuity of direction works first and then symmetry, whereas, normal subjects' completions had one stage, that is, symmetry only.
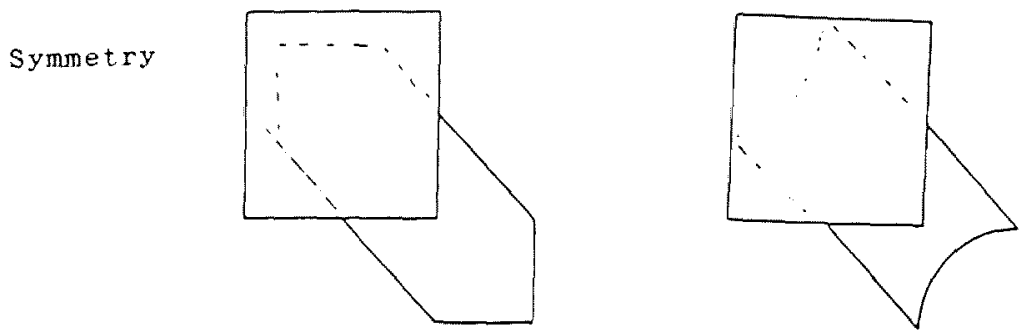

Minimal Distance
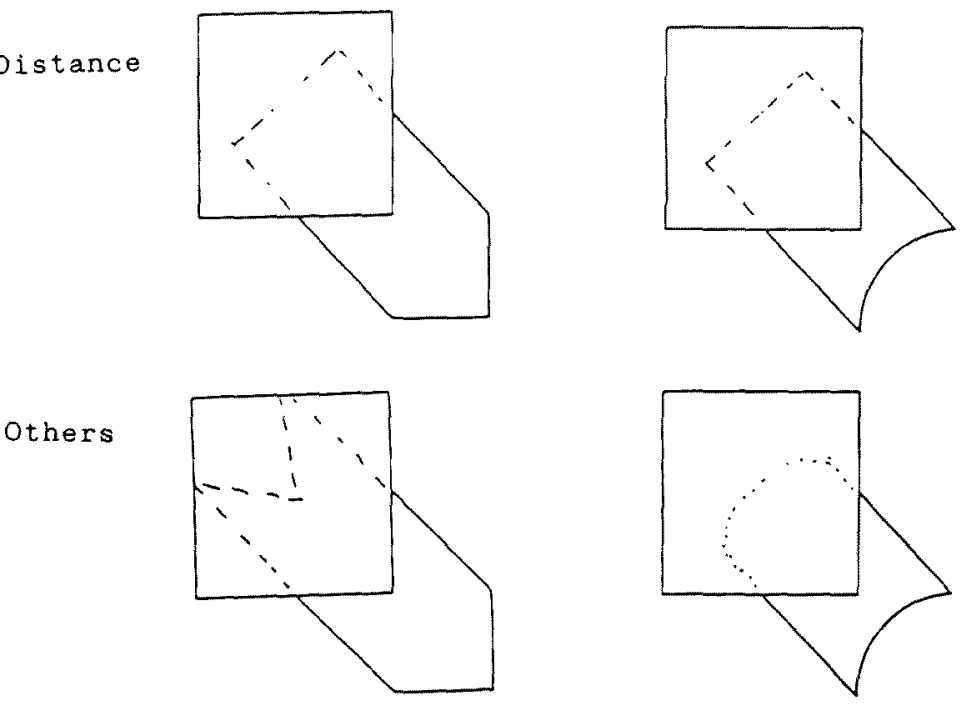

Stimulus e

Stimulus i

Figure 3. Examples of continuity of direction and another principle. 
Table 2

Frequencies of categories in Stimuli e and $\mathrm{i}$ in schizophrenic patients and normals after excluding continuity of direction

\begin{tabular}{ccc}
\hline & Schizophrenic patients & Normals \\
& $N(\%)$ & $N(\%)$ \\
\hline Stimulus e & & \\
Mosaic & $2(6.7)$ & $1(2.0)$ \\
Symmetry & $22(73.3)$ & $40(80.0)$ \\
Minimal distance & $4(13.3)$ & $6(12.0)$ \\
Others & $2(6.7)$ & $3(6.0)$ \\
Stimulus & $3(10.0)$ & $1(2.0)$ \\
Mosaic & $19(63.3)$ & $30(60.0)$ \\
Symmetry & $4(13.3)$ & $9(18.0)$ \\
Minimal distance & $4(13.3)$ & $10(20.0)$ \\
Others & &
\end{tabular}

\section{Discussion}

Only two schizophrenic patients $(6.7 \%)$ perceived a mosaic for all figures. The others perceived a completed object behind an occluding one. Table 1 indicates that either response of continuity of direction or symmetry was dominant in both groups of schizophrenic and normal.

Continuity of direction was the most frequent both in schizophrenics and in normal subjects, except for a few stimuli. This result indicates that amodal completions were based on the Gestalt principle of good continuity for most stimuli in schizophrenics as well as in normals.

Symmetry was the most frequent for Stimulus e in schizophrenic patients and for Stimulus $d$, e and $i$ in normal subjects. Amodal completions were based on symmetry for a few stimuli in both groups. Frequencies of symmetry in schizophrenic patients were significantly lower than those in normal subjects for Stimuli $d, e, g$ and $h$. After excluding the category of continuity of direction for these stimuli, symmetry had nearly the same frequencies in schizophrenic patients as in normal subjects for Stimuli e and $i$. This result suggests that the schizophrenic patients had completed the figures by good continuity first and then by symmetry, whereas the normal subjects had com- pleted by symmetry first of all.

According to Kanizsa (1979), the most frequent completion was continuity of direction for Stimuli a, b, c, d, f, g and h, and minimal distance for Stimuli e and i. However, the latter finding was not completely supported by this study. For Stimulus $d$ in normal subjects, the most frequent completion was symmetry rather than continuity of direction. For Stimuli e and $i$ in both groups, the most frequent completion was symmetry rather than minimal distance. Thus, minimal distance was rather rare in this study, inconsistently with Kanizsa. This means that amodal completion is based on the principle of good continuity or good figure in both groups, while schizophrenic patients showed amodal completion by the principle of good continuity more frequently than normal subjects did.

Why did inter-group differences appear? This problem may be associated with schizophrenic rigidity of mental set (Weiner, 1973). All stimuli used for this study were printed on a piece of paper. After schizophrenic patients had perceived the first stimuli behind an occluding square by the principle of good continuity, the following stimuli might have been completed by the same principle because of the rigidity of their mental set. However, the frequencies of continuity of direction were different among stimuli. 
Moreover, continuity of direction was rather less frequent than symmetry for Stimulus e. These results do not indicate that a particular mental set was preserved consistently for all stimuli. Schizophrenic patients might change their mental set according to the characteristics of the stimuli.

It should be noted that schizophrenic patients showed continuity of direction more frequently than did normal subjects. Continuity of direction was determined by the perception of line directions at the crossing points. Therefore, it was enough to perceive around the crossing points, that is, a part of figure. For continuity of direction, subjects need not perceive the whole figure. On the other hand, it was necessary for symmetry to perceive the whole figure. Generally speaking, global information was processed more primarily than partial in normal subjects (Navon, 1977). Symmetry was determined by perception of a whole figure. This is a reason why normal subjects completed figures by symmetry.

Recent studies have found that schizophrenic information processing is different from that in normal subjects. For example, eye movements in schizophrenic patients were, in these studies, fixated in a narrower range than in normal subjects (Obayashi, Matsushima, Ando, Sakurada, Nakajima, Ando, \& Kojima, 1991; Matsui, Yuasa, \& Kurachi, 1993). Thus, schizophrenic patients were able to search for only a part of a target. This was supported by self-reports of schizophrenics. Schizophrenic patients reported that when they want to perceive the whole face of a doctor, they could perceive only a part of the face, that is, an eye, a mouth and so on, in isolation and fragmentarily (Arieti, 1962). Similar phenomena have been reported in several studies (Bemporad, 1967; Shakow, 1979). These studies indicate that schizophrenic patients did not succeed in perceiving the whole target but only parts of the target. Schizophrenic patients might focus on part of a figure because of perceptual disorder. When the part of a figure that was focussed on was around the crossing point, the Gestalt principle of good continuity might be enough for completion. This might be a reason why schizophrenic patients complete figures by continuity of direction more frequently than normal subjects. After recognizing that a closed figure is not completed by continuity of direction, schizophrenic patients will attempt to use the another principle of good figure, that is, symmetry.

In summary, schizophrenic patients primarily complete a figure by partial information at the crossing points (continuity of direction), and secondly whole information (e.g. symmetry); on the other hand, normals complete a figure by whole information more frequently.

\section{References}

American Psychiatric Association 1987 Diagnostic and statistical manual of mental disorder. 3 rd ed. revised. Washington, DC: Author.

Arieti, S. 1962 The microgeny of thought and perception: A psychiatric contribution. Archives of General Psychiatry, 6, 454-468.

Bemporad, J. R. 1967 Perceptual disorders in schizophrenia. American Journal of Psychiatry, $123,971-976$.

Crookes, T. G., \& Hutt, S. J. 1970 Perception of hidden figures by neurotic and schizophrenic patients. British Journal of Psychiatry, 116, 335 336.

Kanizsa, G. 1979 Organization in vision: Essays on Gestalt perception. New York: Praeger.

Matsui, M., Yuasa, S., \& Kurachi, M. 1993 Cognitive function in patients with schizophrenia. Japanese Journal of Neuropsychology, 9 , 47-54. (In Japanese with English summary)

Mikami, N. 1979a The analysis of schizophrenic drawings using synthetic House-Tree-Person technique: A statistical comparison with normal adults. Japanese Journal of Clinical Psychiatry, 8, 79-90. (In Japanese)

Mikami, N. $1979 \mathrm{~b}$ The analysis of schizophrenic drawings using synthetic House-Tree-Person 
technique: The temporal changes with the condition of schizophrenics. Japanese Journal of Clinical Psychiatr, 8, 1479-1487. (In Japanese)

Miyazaki, T., Hujii, J., \& Kobayashi. A. 1987 A factor analysis of the Koch's Tree-drawing Test: An extraction of three factors from schizophrenic materials. Journal of Japanese Clinical Psychology, 5, 44-50. (In Japanese)

Morita. H. 1989 Features of drawings of schizophrenic patients in synthetic HTP technique. Journal of Japanese Clinical Psychology, 6, 29 39. (In Japanese with English summary)

Nakai. H. 1970 Psychopathology of schizophrenics as revealed by various drawing techniques invented for psychotherapy. Art Therapy, 2, 7790. (In Japanese)

Navon. D. 1977 Forest before trees: The precedence of global features in visual perception. Cognitive Psychology, 9, 353-383.

Obayashi, S., Matsushima, E., Ando, H., Sakurada, M., Nakajima, K., Ando, K., \& Kojima, T. 1991 Eye movements during Benton visual retention test: A comparison between schizophrenics and normal controls. Japanese Journal of Clinical Psychiary, 20, 1255-1261. (In Japanese)

Shakow, D. 1979 Adaptation in schizophrenia: The theory of segmental set. New York: John Wiley \& Sons.

Weckowicz, T. E. 1960 Perception of hidden pictures by schizophrenic patients. Archives of General Psychiatry, 2, $521-527$.

Weiner, I. B. 1966 Psychodiagnosis in schizophrenia. New York: John Wiley \& Sons

Yokota, M. 1992 Schizophrenic patients fail to represent perspectively. Clinical Psychiatry, 34, 238-245. (In Japanese)

Yokota, M. 1993 Global characteristics in drawings of schizophrenic patients on the Kusamura Test. Clinical Psychiatry, 35, 27-33. (In Japanese)

Yokota, M., Yoda, S., Miyanaga. K., Takahashi, S., \& Machiyama, Y. 1986 Constructive disorders in drawing by chronic schizophrenic patients. Clinical Psychiatry, 28, 621-627. (In Japanese) (Received Nov. 29, 1993; accepted May 7, 1994) 